Preprint typeset in JHEP style - HYPER VERSION

CCTP-2013-16

CCQCN-2013-11

CERN-PH-TH/2013-265

KCL-MTH-13-10

\title{
Holographic RG Flows and Nearly-Marginal Operators
}

\section{Jun Bourdier ${ }^{a}$ and Elias Kiritsis ${ }^{b, c, d}$}

${ }^{a}$ Department of Mathematics, King's College London, The Strand, WC2R 2LS, London, United-Kingdom

$b$ APC, Université Paris 7, Diderot, CNRS/IN2P3, CEA/IRFU, Obs. de Paris, Sorbonne Paris Cité, Bâtiment Condorcet, F-75205, Paris Cedex 13, France (UMR du CNRS 7164)

c Theory Group, Physics Department, CERM, CH-1211, Geneva 23, Switzerland

${ }^{d}$ Crete Center for Theoretical Physics, Department of Physics, University of Crete, 71003 Heraklion, Greece

ABSTRACT: The holographic renormalization group flows associated with marginally relevant operators are analysed. The associated perturbative and non-perturbative $\beta$-functions are calculated and the consistent scalar potentials are identified. The presence of a Zamolodchikov metric in the multiscalar case is shown to affect $\beta$ functions starting at two loops.

KEYWORDS: AdS/CFT, holography, strong coupling, renormalization group, marginal operators, non-perturbative $\beta$-functions. 


\section{Contents}

1. Introduction and Results 1

2. Generic Perturbations Near the AdS Boundary 3

2.1 The Standard Perturbative Method 1

2.2 The Superpotential Method 6

2.3 Perturbative Calculation of the Superpotential 7

2.4 The Holographic $\beta$-function 8

2.5 Regularity of the Solutions 8

3. Marginal Operators: The Cubic and Higher Orders Cases 10

4. Marginal Operators: Asymptotically Soft Fixed Points 14

5. The Multiscalar Case 17

6. Acknowledgements 20

Appendix 21

A. Perturbative Calculations for $n=3,4,5$ 21

A.1 $n=3 \quad 21$

A.2 $n=4$

A.3 $n=5 \quad 22$

References 23

\section{Introduction and Results}

The holographic correspondence has revolutionized the way we think about both quantum field theory and string theory. Moreover, it has indicated that the two are intimately related, and that string theory can be viewed as the dynamics of sources of quantum field theory (QFT), [1, 2, 3], namely a form of quantum renormalization group.

A particular item of the QFT tool box, renormalization group (RG) flows have an elegant description in the string theory/gravity language. They correspond to bulk solutions of the equations of motion with appropriate boundary conditions. There 
is a way of writing the bulk equations using the Hamilton-Jacobi formalism, so that

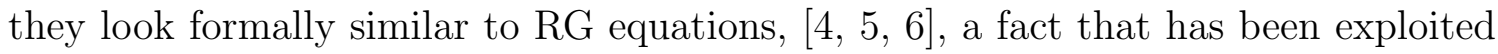
in numerous situations. There have been various takes on the form of the RG/coarse graining procedure in the holographic case, [7], including the Wilsonian approach to IR physics [8].

It is not yet known how to generalize the Hamilton-Jacobi formalism to cases where we are discussing non-trivial states in QFT, namely states with non-trivial temperature and finite density. However, even in that case there are other techniques that allow the calculation of effective potentials, [9], and effective actions, [11], along with the related $R G$ equations.

An important set of flows in QFT are generated by nearly marginal (marginally relevant) operators. This is the example of QCD, as well as other asymptotically free QFTs at zero or finite density (like the Kondo problem). There have been essentially very few investigations of such flows in the literature, [12, 13], because they are not generic in top-down models, and they are explicitly avoided (because of their computational difficulty) in bottom up models. The only examples that have been studied do not seem to be exhaustive. They include, asymptotically-soft AdS minima of the potential, relevant for holographic models of YM, [12], that were argued recently to also be relevant in the cosmology of primordial inflation, [14], and marginally relevant asymptotics of holographic Lifshitz fixed points, [13].

The purpose of this note is to analyze marginally relevant holographic flows in their full generality as they can arise in different contexts, under distinct realizations. They will also be compared to their field theory counterparts.

Moreover, the holographic approach allows the incorporation of non-perturbative corrections to the $\beta$-functions as well as the flow, due to non-trivial vacuum expectation values. We will calculate their form in full generality.

Our results are as follows:

1. We review the case of relevant operators, and the superpotential formalism in order to establish notations.

2. We show that the geometric singularities of a solution correspond to places where the potential $V$ and/or the superpotential $W$ diverge.

3. We define the holographic $\beta$-function, and derive a non-linear first-order equation for it, valid to leading order at strong coupling and large $-N$.

4. We analyze the case of classically marginal operators whose potential has zero mass term and the first non-trivial term is $\phi^{n}, n \geq 3$ in the regime $\phi \rightarrow 0$. The solutions around the UV fixed point $(\phi=0)$ are derived and indicate that such operators have a perturbative $\beta$-function that starts at $n-2$ loop order. 
5. We find the leading non-perturbative corrections, that behave exponentially in the inverse of the coupling constant.

6. We find however that the scalar diverges as it approaches the boundary, and this case seems inconsistent with the original assumptions $(\phi \ll 1)$.

7. We also analyze classical marginal operators near a UV fixed point at $\phi \rightarrow$ $\pm \infty$. This case corresponds to asymptotically-soft fixed points advocated for IHQCD, [12], and appears also in cosmology in Starobinsky-like models, [15, 14. We find that they have always a non-trivial one-loop $\beta$-function, in close analogy to YM in four dimensions. Moreover, the scalar coupling asymptotes properly to the UV fixed point at the boundary of the geometry.

8. The leading non-perturbative corrections to the $\beta$-function are evaluated and shown to be similar to those expected in YM.

9. The multiscalar case is analyzed in a similar spirit. Here there is also a Zamolodchikov metric that enters the bulk description apart from the scalar potential. The perturbative RG flows are constructed near a UV fixed point, and it is shown that the contributions of the Zamolodchikov metric to the $\beta$-functions appear first at two-loops.

We conclude with an important open question to which we know the answer only in very special cases : which concrete cases of marginal operators arise from top-down string theory constructions at string tree level?

\section{Generic Perturbations Near the AdS Boundary}

We will start our study by setting up the general problem of RG flows of a scalar operator. To do this we will have to confine ourselves to Einstein-Dilaton (ED) theory which at the two derivative level ${ }^{1}$ after field redefinitions, takes the form:

$$
S_{2 d}=M^{d-1} \int d^{d+1} x \sqrt{-g}\left\{R-\frac{1}{2} \partial_{\mu} \phi \partial^{\mu} \phi+V(\phi)\right\}
$$

Here $d$ is the space-time dimension of the QFT (living on the boundary), while the space-time dimension of the bulk ED gravity is $d+1$.

The metric as usual is dual to the stress tensor in the QFT and is always included, as it is always sourced even in the vacuum saddle-point (gravitational) solution ${ }^{2}$. We also have included a bulk scalar field $\phi$ dual to a scalar operator in the QFT.

\footnotetext{
${ }^{1}$ Generically this is justified at the strong coupling and large $\mathrm{N}$ limit, however adding higher derivatives of the scalar and the metric, does not affect the structure of the $\beta$ functions in the scaling region.

${ }^{2}$ The basic rules of the holographic correspondence are detailed in several papers and textbooks, see eg. [16].
} 
In this section, there are no restrictions on the form of the potential, apart from the requirement of at least one extremum at $\phi=\phi_{*}$ which yields an $A d S_{d+1}$ geometry as the vacuum solution, with curvature scale $\ell$ and signals the existence of a CFT:

$$
V\left(\phi_{*}\right)=\frac{d(d-1)}{\ell^{2}}+\cdots
$$

The general form of the equations of motion obtained from (2.1) is then:

$$
\begin{aligned}
& \nabla^{\mu} \nabla_{\mu} \phi+V^{\prime}(\phi)=0 \\
& R_{\mu \nu}-\frac{1}{2} g_{\mu \nu}\left(R-\frac{1}{2}(\partial \phi)^{2}+V(\phi)\right)-\frac{1}{2} \partial_{\mu} \phi \partial_{\nu} \phi=0 .
\end{aligned}
$$

\subsection{The Standard Perturbative Method}

We will proceed now to study RG flows in the neighborhood of the AdS fixed point, (2.2), also known in QFT as the scaling region.

In the conformal coordinate frame for the metric, the ansatz that we will be studying for the Poincaré-invariant field theory metric and the dilaton is:

$$
d s^{2}=e^{2 A(r)}\left[d r^{2}+d x_{\mu} d x^{\mu}\right] \quad, \quad \phi(r) .
$$

were the radial direction is the holographic direction and will be related to the RG scale as is standard in holography.

The $A d S_{d+1}$ solution:

$$
e^{2 A}=\frac{\ell^{2}}{r^{2}} \quad, \quad \phi=0,
$$

then corresponds to the (UV) CFT, with the boundary at $r=0$. Substituting the ansatz (2.5) into (2.3) and (2.4), we obtain the equations for the scale factor and the scalar:

$$
\begin{aligned}
& (d-1)\left[A^{\prime}(r)^{2}-A^{\prime \prime}(r)\right]-\frac{1}{2} \phi^{\prime}(r)^{2}=0, \\
& (d-1) A^{\prime \prime}(r)+(d-1)^{2} A^{\prime}(r)^{2}-e^{2 A(r)} V(\phi)=0 \\
& e^{-2 A(r)}\left[\phi^{\prime \prime}(r)+(d-1) A^{\prime}(r) \phi^{\prime}(r)\right]+\frac{d V(\phi)}{d \phi}=0,
\end{aligned}
$$

where we have used the convention ' $\equiv \frac{d}{d r}$. These equations capture the solutions with Poincaré invariance in $d$ dimensions.

Equations (2.7)-(2.9) can be solved perturbatively near the boundary. We choose the UV minimum to be at $\phi_{*}=0$ without loss of generality ${ }^{3}$. We then expand the

\footnotetext{
${ }^{3}$ It can be made to vanish by a shift in $\phi$ that does not affect the other terms in the action.
} 
potential near the minimum to quadratic order ${ }^{4}$ :

$$
V(\phi)=\frac{d(d-1)}{\ell^{2}}+\frac{1}{2} m^{2} \phi^{2}+\cdots \quad, \quad \phi \rightarrow 0
$$

Working at next to leading order, we obtain:

$$
\begin{aligned}
& \phi(r)=c_{1} r^{\Delta_{+}}+c_{2} r^{\Delta_{-}}+\cdots, \Delta_{ \pm}=\frac{d \pm \sqrt{d^{2}-4 m^{2} \ell^{2}}}{2} \\
& A(r)=\log \frac{\ell}{r}-\frac{c_{2}^{2} \Delta_{-} r^{2 \Delta_{-}}}{4(d-1)\left(1+2 \Delta_{-}\right)}-\frac{c_{1}^{2} \Delta_{+} r^{2 \Delta_{+}}}{4(d-1)\left(1+2 \Delta_{+}\right)}-\frac{c_{1} c_{2} \Delta_{+} \Delta_{-} r^{d}}{d\left(d^{2}-1\right)}+\cdots
\end{aligned}
$$

with $c_{1,2}$ real.

We therefore derive the basic rule of the $A d S / C F T$ dictionary, namely that

$$
m^{2} \ell^{2}=\Delta_{+} \Delta_{-}=\Delta(d-\Delta)
$$

where $\Delta$ is the scaling dimension of the operator $\mathcal{O}$ dual to $\phi$ in the dual QFT.

There are several cases to be distinguished depending on the values of the mass:

1. $\frac{d^{2}}{4}>m^{2} \ell^{2}>0$ corresponds to relevant operators. If $m^{2} \ell^{2}>\frac{d^{2}}{4}$, the dimensions $\Delta_{ \pm}$are complex (and therefore this case is unacceptable). The scalar violates the BF bound.

There are two important subcases:

- $0<m^{2} \ell^{2}<\frac{d^{2}}{4}-1$ in which only $\Delta=\Delta_{+}$and $c_{1}$ is the vev while $c_{2}$ is the source in (2.11).

- $\frac{d^{2}}{4}>m^{2} \ell^{2}>\frac{d^{2}}{4}-1$, where there are two quantizations of the scalar field as both linearly independent solutions are normalizable near the boundary. In one, $\Delta=\Delta_{+}$and $c_{1,2}$ are as above, while in the other, $\Delta=\Delta_{-}$and the role of $c_{1,2}$ is reversed.

2. $m^{2}<0$ corresponds to irrelevant operators.

3. $m=0$ corresponds to operators that to this order are marginal.

\footnotetext{
${ }^{4}$ The convention chosen here for the mass term of the potential differs from [10]. There it appears with a minus sign.
} 


\subsection{The Superpotential Method}

There is however a more convenient formalism for our purposes, [12, 9], which uses a different set of coordinates, the domain wall coordinates. This is convenient because we will write the radial evolution equations for the scalar as first order RG equations in terms of a $\beta$-function.

We use a new bulk coordinate $u$, related to the previous one through the relation $r=\ell e^{-u / \ell}$ in the $A d S$ case. Hence the boundary of an asymptotically $A d S$ solution now lies at $u=+\infty$. Using these coordinates, the ansatz (2.5) for the metric takes the form:

$$
d s^{2}=d u^{2}+e^{2 A(u)} d x_{\mu} d x^{\mu} .
$$

The $A d S_{d+1}$ solution is now:

$$
A(u)=\frac{u}{\ell}, \phi(u)=0
$$

Substituting the new form of the ansatz into the equations of motion (2.3) and (2.4), we obtain:

$$
\begin{aligned}
& d(d-1) \dot{A}(u)^{2}-\frac{1}{2} \dot{\phi}^{2}-V(\phi)=0, \\
& 2(d-1) \ddot{A}(u)+\dot{\phi}(u)^{2}=0 \\
& \ddot{\phi}(u)+d \dot{A}(u) \dot{\phi}(u)+\frac{d V(\phi)}{d \phi}=0 .
\end{aligned}
$$

We then introduce a function $W(\phi)$ that we call the superpotential ${ }^{5}$. It is a function of the scalar only but does not depend explicitly on the radial coordinate. The superpotential satisfies:

$$
\frac{d W^{2}}{2(d-1)}-W^{\prime 2}=2 V(\phi) .
$$

where the prime is derivative with respect to $\phi$. If (2.19) is valid then the first order equations:

$$
\dot{A}=-\frac{W}{2(d-1)} \quad, \quad \dot{\phi}=\frac{d W}{d \phi} \equiv W^{\prime}
$$

are equivalent to the equations of motion (2.16), (2.17) and (2.18).

The equations (2.20) are first order equations. Hence we only need one initial condition for each function $A$ and $\phi$. However, $W$ is determined through (2.19) which is also a a first order differential equation, and we therefore need one initial condition for $W$ to solve the equation uniquely. In total we have 3 initial conditions for this system of equations.

\footnotetext{
${ }^{5}$ In supergravity actions it is indeed the superpotential, but it is a more general concept, and as shown in [9] it gives the boundary QFT effective potential for the scalar.
} 
This is the correct number of the initial conditions of the original set of equations (2.16)-(2.18) as the $\phi$ equation is second order and has two arbitrary initial conditions while the equation for $A$ is first order, and has another integration constant.

The non-trivial integration constant of equation (2.19) controls the (non-perturbative) expectation value of the operator dual to $\phi$. Typically, as the equation (2.19) is nonlinear, the space of solutions consists of two generic families that lead to singular solutions for the scalar, and a unique solution in between that gives a regular evolution for the scalar, [18, 19].

\subsection{Perturbative Calculation of the Superpotential}

The equation (2.19) can be solved perturbatively near the boundary $(\phi \rightarrow 0)$ as a regular expansion in $\phi$ :

$$
W(\phi)=W_{0}+W_{1} \phi+W_{2} \phi^{2}+\cdots .
$$

Expanding to second order and using the potential (2.10), we obtain the following set of equations:

$$
\begin{gathered}
\frac{d W_{0}^{2}}{2(d-1)}-W_{1}^{2}=\frac{2 d(d-1)}{\ell^{2}}, \frac{d W_{1} W_{0}}{(d-1)}-4 W_{1} W_{2}=0, \\
\frac{d W_{1}^{2}}{2(d-1)}+\frac{d W_{0} W_{2}}{d-1}-4 W_{2}^{2}-6 W_{1} W_{3}=m^{2} .
\end{gathered}
$$

which are solved in terms of $W_{0}$ :

$$
W_{1}= \pm \sqrt{\frac{2 d}{\ell^{2}}-\frac{d W_{0}^{2}}{2(d-1)^{2}}} \quad, \quad W_{2}=\frac{d W_{0}}{4(d-1)} .
$$

Hence, at quadratic order the expansion is:

$$
W(\phi)=W_{0} \pm \sqrt{\frac{2 d}{\ell^{2}}-\frac{d W_{0}^{2}}{2(d-1)^{2}}} \phi+\frac{d W_{0}}{4(d-1)} \phi^{2}+O\left(\phi^{3}\right) .
$$

However, this solution is too general for our purposes and does not satisfy important holographic requirements. We need that after solving the first order equations using this superpotential, that we obtain the neighborhood of the boundary. We solve (2.20) using (2.25) to linear order, $W(\phi)=W_{0}+W_{1} \phi$. The equation $\dot{\phi}=W_{1}$ gives:

$$
\phi(u)=W_{1}\left(u-u_{0}\right) .
$$

where we have adjusted the boundary at $u=u_{0}$ so that $\phi$ vanishes there as it should. For the metric, we are led to solve $\dot{A}=-W / 2(d-1)$ which implies

$$
d s^{2}=d u^{2}+e^{A_{0}-\frac{\left(W_{1}^{2}\left(u-u_{0}\right)+W_{0}\right)^{2}}{2(d-1) W_{1}^{2}}} d x^{\mu} d x_{\mu} .
$$


It is clear, that $u=u_{0}$ is not a boundary for this metric (where the scale factor must diverge) unless $W_{1}=0$. Hence we must impose $W_{1}=0$ and the expansion (2.25) is no longer valid. Instead we obtain

$$
W(\phi)=-\frac{2(d-1)}{\ell}-2 \frac{\Delta_{ \pm}}{\ell} \phi^{2}+O\left(\phi^{3}\right) .
$$

Both overall signs for $W$ solve the equations. The minus sign corresponds to leaving a UV Fixed point. This can be seen from (2.20), and the fact that $u$ decreases away from the boundary at $u=+\infty$ and the fact that $A$ should decrease.

\subsection{The Holographic $\beta$-function}

In holography with a two-derivative bulk action, the scale factor $e^{A}$ plays the role of an RG scale. Apart from the fact that near the AdS boundary $r \rightarrow 0$ (or $u \rightarrow+\infty)$ it coincides with the radial coordinate, it can be shown from the equations (2.16)(2.18) that it is a monotonic function, that diverges in the UV and vanishes in the IR, [17]. We may therefore associate the exponent of the scale factor $A \leftrightarrow \log \mu$ to the logarithm of the RG scale.

In view of this we may define the holographic analogue of the $\beta$ function as

$$
\frac{d \phi}{d A} \equiv \beta_{\phi}=-\frac{2(d-1)}{W} \frac{d}{d \phi} W(\phi),
$$

where we have used the first order equations of motion (2.20) in the righthand side above.

The nonlinear equation for the superpotential (2.19) implies a non-linear equation for the $\beta$-function. Differentiating (2.19) and using (2.20), we obtain a first order non-linear differential equation for the holographic $\beta$-function:

$$
\beta=(d-1) \frac{d}{d \phi} \log \left(\frac{\beta^{2}-2 d(d-1)}{V}\right) .
$$

\subsection{Regularity of the Solutions}

Taking $\phi$ as a coordinate instead of $u$, the metric becomes:

$$
d s^{2}=\frac{d \phi^{2}}{W^{\prime 2}}+e^{2 A}\left(d x_{\mu} d x^{\mu}\right),
$$

where we have used the equations of motion (2.20). The Ricci scalar can be computed to be

$$
R=\frac{d}{4(d-1)}\left[W^{2}-8 V\right] .
$$

where we have once again used the equations of motion and where all derivatives are with respect to $\phi$. On the other hand, taking the trace of the Einstein equations (2.4) yields:

$$
R=\frac{1}{2}(\partial \phi)^{2}-\frac{d+1}{d-1} V
$$


Substituting this back into the Einstein equations allows us to obtain a simple expression for the Ricci tensor:

$$
R_{\mu \nu}=\frac{1}{2} \partial_{\mu} \phi \partial_{\nu} \phi-\frac{V}{d-1} g_{\mu \nu}
$$

From this we can readily deduce the following invariant:

$$
R_{\mu \nu} R^{\mu \nu}=\frac{d}{16(d-1)^{2}}\left[16(d+1) V^{2}-8 d V W^{2}+d W^{4}\right]
$$

Finally, we can calculate directly the Riemann invariant:

$$
R_{\mu \nu \rho \sigma} R^{\mu \nu \rho \sigma}=\frac{d}{8(d-1)^{3}}\left[32(d-1) V^{2}-16(d-1) V W^{2}+(2 d-1) W^{4}\right] .
$$

From these three formulae (2.32), (2.35) and (2.36) we can conclude the following concerning the regularity of the metric (finiteness of the curvature invariants):

1. If at a point $\phi$ both $\mathrm{V}$ and $\mathrm{W}$ are finite, then the geometry is regular.

2. If at a point $\phi, V$ diverges but $W$ remains bounded, or vice versa, this is a curvature singularity.

3. If $V \rightarrow \infty$ as $\phi \rightarrow \phi_{*}$, then in order for the scalar curvature to be regular, $W$ should also diverge as $W^{2} \sim 8 V$. However in that case the Ricci square is always divergent.

We conclude that the geometric singularities correspond to places where $V$ and/or $W$ diverge.

If we now assume that we have an Einstein space, i.e. that $R_{\mu \nu}=\Lambda(\phi) g_{\mu \nu}$, then from (2.32) and (2.35) we obtain:

$$
\begin{aligned}
& \Lambda^{2}(\phi)(d+1)=\frac{d}{16(d-1)^{2}}\left[16(d+1) V^{2}-8 d V W^{2}+d W^{4}\right] \\
& \Lambda(\phi)(d+1)=\frac{d}{4(d-1)}\left[W^{2}-8 V\right] .
\end{aligned}
$$

Eliminating $W$ out from these equations gives:

$$
V(\phi)=-(d-1) \Lambda
$$

In particular in the case of $\operatorname{AdS}$ space $\Lambda$ is constant and then the potential must be constant. 


\section{Marginal Operators: The Cubic and Higher Orders Cases}

In this section we will assume that the extremal point of the potential is degenerate, $m=0$ and therefore the operator is marginal to leading order. We will also assume that the first non-trivial derivative comes in at order $n \geq 3$.

We use the superpotential method of section 2.2 to study the gravitational solutions with the following potential:

$$
V(\phi)=\frac{d(d-1)}{\ell^{2}}+\frac{g_{n}}{\ell^{2}} \phi^{n}+\cdots,
$$

near $\phi \rightarrow 0$ where we have chosen the critical point of this potential to be.

The equation (2.19) can be solved perturbatively near the boundary by expanding $W$ around the $A d S$ solution $\phi=0$ :

$$
W(\phi)=\sum_{n=0}^{\infty} W_{n} \phi^{n}
$$

We obtain ${ }^{6}$ :

$$
W(\phi)=-\frac{1}{\ell}\left(2(d-1)+\frac{g_{n}}{d} \phi^{n}+\frac{g_{n}^{2} n^{2}}{2 d^{3}} \phi^{2 n-2}+\delta_{n, 3} \frac{54 g_{n}^{3}}{d^{5}} \phi^{2 n-1}\right)+O\left(\phi^{2 n}\right) .
$$

We may now use the superpotential found, to compute the (perturbative) $\beta$ function as

$$
\beta_{\phi} \equiv \frac{d \phi}{d A}=-\frac{2(d-1)}{W} \frac{d}{d \phi} W(\phi) .
$$

Using the perturbative solution (3.3) for $W$, we find to next to leading order:

$$
\beta_{\phi}=-\frac{g_{n} n}{d} \phi^{n-1}-\frac{g_{n}^{2} n^{2}(n-1)}{d^{3}} \phi^{2 n-3}+O\left(\phi^{2 n-1}\right) .
$$

From (3.5) we can interpret this $\beta$ function as follows. If $n=2$, at lowest order, we obtain a linear term in $\phi$, proportional to $d-\Delta$. This the standard classical contribution to the $\beta$-function of non-marginal operators.

Marginal operators have no quadratic term around the fixed point. If the first non-trivial term is cubic $(n=3)$, then the leading $\beta$ function is quadratic and this is similar to the QFT case where a one-loop contribution is the leading contribution to the $\beta$-function.

When $n>3$, then this is similar to the field theory case, where the first nontrivial contribution arises at $n-2$ loops. We also observe, that a single bulk $\phi^{n}$ interaction, generates an infinite number of terms in the perturbative $\beta$ functions as seen in (3.5). For $n=3$, all higher terms appear, while for higher $n$ there are gaps at

\footnotetext{
${ }^{6} \mathrm{We}$ assume here the presence only of the $\phi^{n}$ term in the potential in order to track which terms in the $\beta$-function are affected.
} 
low number of loops. This is explicitly seen in appendix A where the superpotential and the $\beta$ function are computed to higher orders.

The standard perturbative method can be used to obtain explicit expressions for the metric ${ }^{7}$ and scalar field. For a dual operator $\mathcal{O}$ with $\langle\mathcal{O}\rangle=0$, we obtain:

$$
\phi(A)=\phi_{0}-\frac{g_{n} n}{d} A \phi_{0}^{n-1}-\frac{g_{n}^{2} n^{2}(n-1)}{d^{2}} A\left(\frac{1}{d}-\frac{1}{2} A\right) \phi_{0}^{2 n-3}+O\left(\phi_{0}^{2 n-1}\right),
$$

where $\phi_{0}$ is the infinitesimal source for $\phi$. We can also express the scale factor in terms of the radial coordinate $r$ :

$$
A(r)=\log \frac{\ell}{r}-\frac{g_{n}}{2 d(d-1)} \phi_{0}^{n}+\frac{g_{n}^{2} n^{2}}{4(d-1) d^{3}}\left[1-2 d\left(1-\log \frac{r}{\ell}\right)\right] \phi_{0}^{2 n-2}+O\left(\phi_{0}^{2 n-1}\right) .
$$

\section{Non-perturbative Corrections}

We will now try to extend this perturbative solution by including non-perturbative terms that originate in non-trivial vevs for the bulk scalar. In the case of a massive scalar field, it is known that the full non-perturbative solution is expressed as follows, [9] :

$$
W(\phi)=\sum_{k=0}^{\infty} W_{k}(\phi) \quad, \quad W_{k}(\phi)=\mathcal{C}^{k} \phi^{k \delta} \sum_{j=0}^{\infty} A_{k, j} \phi^{j}
$$

where $\delta$ is equal to $d / \Delta_{-}$, and where $\mathcal{C}$ is related to the vev $\langle\mathcal{O}\rangle . W_{0}(\phi)$ denotes the perturbative power series solution, valid in the $\phi \rightarrow 0$ regime.

For the marginal case, the non-perturbative contributions do not take the form of non-integer powers of $\phi$. To obtain the form of the possible non-perturbative terms, we will expand the superpotential again as in (3.8) and we will treat $W_{k>1}$ as small. This is equivalent to a small vev expansion, and we will formalise it by writing

$$
W=\sum_{k=0}^{\infty} x^{k} W_{k}
$$

and do perturbation theory in $x$ and then set it to one at the end.

Substituting (3.9) into (2.19), and separating powers of $x$, we obtain for any integer $k \geq 0$ :

$$
\frac{d}{2(d-1)} \sum_{i+j=k} W_{i} W_{j}-\sum_{i+j=k} W_{i}^{\prime} W_{j}^{\prime}=\delta_{k, 0} 2 V
$$

At each order, we obtain a first-order linear equation for $W_{k}$ of the form:

$$
\frac{d}{(d-1)} W_{0} W_{k}-2 W_{0}^{\prime} W_{k}^{\prime}=f_{k}(\phi),
$$

\footnotetext{
${ }^{7}$ Here $A$ denotes the logarithm of the scale factor.
} 
where $f_{k}(\phi)$ is a function depending on the $W_{m}$ and $W_{m}^{\prime}$ for $0 \leq m \leq k-1$. The general solution to this equation is:

$$
W_{k}=\exp \left[-d \int \frac{d \phi}{\beta_{0}(\phi)}\right]\left(\mathcal{C}_{k}-\int \frac{f_{k}(\phi)}{2 W_{0}^{\prime}} \exp \left[d \int \frac{d \tilde{\phi}}{\beta_{0}(\tilde{\phi})}\right] d \phi\right) .
$$

For the leading correction $W_{1}$, we obtain (2.19):

$$
\frac{d}{d-1} W_{0} W_{1}-2 W_{0}^{\prime} W_{1}^{\prime}=0
$$

and therefore

$$
W_{1}=\mathcal{C}_{1} \exp \left[-d \int_{0}^{\phi} \frac{d \chi}{\beta_{0}(\chi)}\right]
$$

where we have used the definition of the perturbative holographic $\beta$-function:

$$
\beta_{0}=-2(d-1) \frac{W_{0}^{\prime}}{W_{0}}
$$

The constant $\mathcal{C}_{1}$ is non-perturbative and can be fixed only if a global and regular flow solution for the scalar and the metric has been found. Similarly, for $k=2$, we obtain:

$$
\frac{d}{(d-1)} W_{0} W_{2}-2 W_{0}^{\prime} W_{2}^{\prime}=W_{1}^{\prime 2}-\frac{d}{2(d-1)} W_{1}^{2} \equiv f_{2}
$$

with solution

$$
W_{2}=\exp \left[-d \int_{0}^{\phi} \frac{d \phi}{\beta_{0}(\phi)}\right]\left(\mathcal{C}_{2}+\int_{0}^{\phi}\left(\frac{d}{\beta_{0}^{2}}-\frac{1}{2(d-1)}\right) \frac{d(d-1)}{\beta_{0}} \mathcal{C}_{1}^{2} e^{-\int_{0}^{\phi} \beta_{0}\left(\frac{d}{\beta_{0}^{2}}-\frac{1}{2(d-1)}\right)} d \chi\right)
$$

where we have used:

$$
W_{0}=e^{-\frac{1}{2(d-1)} \int d \phi \beta_{0}(\phi)} \quad, \quad W_{1}=\mathcal{C}_{1} e^{-d \int \frac{d \phi}{\beta_{0}}} \quad, \quad W_{0}^{\prime}=-\frac{\beta_{0}}{2(d-1)} e^{-\frac{1}{2(d-1)} \int d \phi \beta_{0}} .
$$

Note that the integration constant $\mathcal{C}_{2}$ can be absorbed into $\mathcal{C}_{1}$ in $W_{1}$. This is as it should, as the full solution should contain a single undetermined constant as the equation for the superpotential is of the first order.

By induction it is straightforward to see that all the higher terms $W_{i}, i \geq 3$ can also be expressed in terms of integrals of $\beta_{0}$. We will now compute explicitly $W_{1}$, using the perturbative form (3.3) obtained for $W_{0}$. From (3.13), we have:

$$
\frac{d \log W_{1}}{d \phi}=\frac{d}{2(d-1)} \frac{W_{0}}{W_{0}^{\prime}}=\frac{d^{2}}{g_{n} n \phi^{n-1}}-\frac{n-1}{\phi}-\delta_{n, 3} \frac{18 g_{n}}{d^{2}}+O(\phi),
$$

which is solved by:

$$
W_{1}(\phi)=\mathcal{C}_{1} e^{-\frac{d^{2}}{g_{n} n(n-2) \phi^{n-2}}-(n-1) \log \phi-\delta_{n, 3} \frac{18 g_{n}}{d^{2}} \phi+\cdots} .
$$


It should be noted here that the non-perturbative solution is captured completely in the non-linear equation for the $\beta$ function (2.30) we derived earlier. Also the arbitrary integration constant that appears in the non-perturbative part, is eventually fixed by demanding that the superpotential gives rise to a globally regular solution. This constraints the integration constant to take a restricted set of values, typically only one, 9].

We can also compute the contribution $\beta_{1}$ of the non-perturbative part of the superpotential to the $\beta$-function:

$$
\beta=-2(d-1) \frac{d}{d \phi} \log W=\underbrace{-2(d-1) \frac{d}{d \phi} \log W_{0}}_{\beta_{0}} \underbrace{-2(d-1) \frac{d}{d \phi} \frac{W_{1}}{W_{0}}}_{\beta_{1}}+\cdots
$$

with

$$
\beta_{1}=-2(d-1)\left(\frac{W_{1}^{\prime}}{W_{0}}-\frac{W_{0}^{\prime}}{W_{0}} \frac{W_{1}}{W_{0}}\right)=\left(\frac{2 d(d-1)}{\beta_{0}}-\beta_{0}\right) \frac{\mathcal{C}_{1}}{W_{0}} e^{-d \int \frac{d \phi}{\beta_{0}}} .
$$

We obtain:

$$
\begin{aligned}
\beta_{1}= & \ell \mathcal{C}_{1} e^{-\frac{d^{2}}{g_{n} n(n-2) \phi^{n-2}}-(n-1) \log \phi-\delta_{n, 3} \frac{18 g_{n}}{d^{2}} \phi} \\
& \times\left(\frac{d^{2}}{g_{n} n \phi^{n-1}}-\frac{n-1}{\phi}-\delta_{n, 3} \frac{18 g_{n}}{d^{2}}+O(\phi)\right) .
\end{aligned}
$$

Therefore for $n=3$ corresponding to a one-loop $\beta$-function we obtain

$$
\beta_{1} \simeq \mathcal{C} \frac{d^{2}}{3 g_{3} \phi^{2}} e^{-\frac{d^{2}}{3 g_{3} \phi}}
$$

which has the typical non-perturbative form we are familiar with from QCD.

\section{Asymptotic Behavior of the Coupling}

We can now use the expression for $\beta_{\phi}$ to discuss the asymptotic behaviors depending on the coupling constant $g_{n}$. For this, we integrate (3.5) in the following way:

$$
\int_{\phi\left(u_{0}\right)}^{\phi(u)} \frac{d \phi}{\beta_{\phi}}=\int_{A\left(u_{0}\right)}^{A(u)} d A
$$

where $u_{0}$ is some arbitrary scale, and which then gives:

$$
-\frac{d}{g_{n} n} \int_{\phi\left(u_{0}\right)}^{\phi(u)} \frac{1}{\phi^{n-1}}\left(1-\frac{g_{n} n(n-1)}{d} \phi^{n-2}+O\left(\phi^{n}\right)\right) d \phi=A(u)-A\left(u_{0}\right) .
$$

For $n>2$, the result is:

$\frac{d}{g_{n} n(n-2)}\left(\frac{1}{\phi^{n-2}(u)}-\frac{1}{\phi^{n-2}\left(u_{0}\right)}\right)+(n-1) \log \left(\frac{\phi(u)}{\phi\left(u_{0}\right)}\right)+O\left(\phi^{2}\right)=A(u)-A\left(u_{0}\right)$. 
Since the UV (or near-boundary limit) limit is at $u \rightarrow+\infty$ and $A(u) \sim u / \ell$, we observe that the theory is asymptotically free for $g_{n}>0$. The IR limit is at $u \rightarrow-\infty$ and this time the theory will be IR free for $g_{n}<0$.

Inverting the above formula, one obtains:

$$
\phi \simeq \phi_{0}-\frac{g_{n} n}{d} \phi_{0}^{n-1} A+\frac{g_{n}^{2} n^{2}(n-1) \phi_{0}^{2 n-3}}{2 d^{2}}\left(A^{2}+\mathcal{O}(A)\right)+\mathcal{O}\left(\phi_{0}^{2 n-2}\right),
$$

in agreement with (3.6).

We summarize the solution as follows: we have set our UV fixed point to $\phi=0$ without loss of generality, and expanded the potential near this fixed point, with $\phi \ll 1$. We have found that near the boundary, $A \rightarrow \infty$, the solution for $\phi$ is given by (3.6) or (3.28) and therefore, $\phi \rightarrow \infty$ as we approach the boundary, $A \rightarrow \infty$. This contradicts our assumptions and therefore this case is inconsistent.

\section{Single-term $\beta$-function}

We have seen that a single non-trivial term in the bulk scalar potential produces an infinite number of terms in the $\beta$ function. In this part we will ask the opposite question: are there bulk potentials that give a (perturbative) $\beta$ function containing as single term?

This is equivalent to imposing:

$$
-2(d-1) \frac{W^{\prime}}{W}=-k \phi^{n-1}
$$

which can be solved by :

$$
W(\phi)=\mathcal{C} e^{\frac{k}{2 n(d-1)} \phi^{n}},
$$

where $\mathcal{C}$ is an integration constant. Substituting this result back into (2.19), we get:

$$
\begin{aligned}
V(\phi) & =\frac{1}{2}\left(\frac{d}{2(d-1)}\left(\mathcal{C} e^{\frac{k}{2 n(d-1)} \phi^{n}}\right)^{2}-\left(\mathcal{C} \frac{k}{2(d-1)} \phi^{n-1} e^{\frac{k}{2 n(d-1)} \phi^{n}}\right)^{2}\right) \\
& =\frac{1}{2} \mathcal{C}^{2} e^{\frac{k}{n(d-1)} \phi^{n}}\left(\frac{d}{2(d-1)}-\frac{k^{2}}{4(d-1)^{2}} \phi^{2 n-2}\right)
\end{aligned}
$$

The potential therefore has Liouville-type interactions.

\section{Marginal Operators: Asymptotically Soft Fixed Points}

In our previous discussion we have assumed that the UV fixed point is a finite value of the scalar field. This is not the only option, and in this section we will describe the only alternative, namely asymptotically soft nearly marginal fixed points. This 
is the case utilized in holographic models of YM like Improved Holographic QCD (IHQCD), 12, 20], and is also relevant in cosmology (see [14]).

The critical point now will be at $\phi \rightarrow \pm \infty$. Without loss of generality we consider the following potential:

$$
V(\phi)=\frac{d(d-1)}{\ell^{2}}\left[1+\sum_{n=1}^{\infty} V_{n} \lambda^{n}\right] \quad, \quad \lambda \equiv e^{-a \phi} \quad, \quad a>0
$$

and we will be working in the region $\phi \rightarrow \infty$, so that $\lambda \rightarrow 0 . \quad \lambda=0$ is an AdS fixed point. This potential has the property that around the AdS fixed point, all derivatives of the potential vanish justifying the term asymptotically soft fixed-point.

Solving perturbatively (2.19), we obtain for the first few coefficients:

$$
\begin{gathered}
W=\sum_{n=0}^{\infty} W_{n} \lambda^{n} \quad, \quad W_{0}=-\frac{2(d-1)}{\ell}, \quad W_{1}=W_{0} \frac{V_{1}}{2}, \\
W_{2}=W_{0}\left(\frac{V_{2}}{2}-\left[1-\frac{2(d-1) a^{2}}{d}\right] \frac{V_{1}^{2}}{8}\right),
\end{gathered}
$$

where once again the sign of $W_{0}$ has been chosen so that the solution leads to an $A d S$ boundary.

The $\beta$-function is calculated as follows:

$$
\beta_{\lambda} \equiv \frac{d \lambda}{d A}=-a \lambda \frac{d \phi}{d A}=2(d-1) a \lambda \frac{W^{\prime}}{W} .
$$

Substituting the previous results found for the superpotential, we obtain:

$$
\beta_{\lambda}=-(d-1) a^{2} V_{1} \lambda^{2}-2(d-1) a^{2}\left[V_{2}-\left[1-\frac{2(d-1) a^{2}}{d}\right] \frac{V_{1}^{2}}{2}\right] \lambda^{3}+\ldots
$$

This is again a $\beta$-function corresponding to a marginal coupling, with the difference that here there is no option on what is the leading term in the $\beta$ function. It always is the "one-loop" term.

From here, we can compute the corrections to the metric and to the dilaton by solving (2.20). For the dilaton we obtain

$$
\dot{\phi}=-\frac{1}{a \lambda} \frac{d \lambda}{d u}=-a W_{1} \lambda-2 a W_{2} \lambda^{2}+\ldots,
$$

which is solved by:

$$
\lambda(u)=\frac{\ell}{a^{2}(d-1) V_{1} u}+\frac{\ell^{2}}{a^{4}(d-1)^{2} V_{1}}\left(\left[1-\frac{2(d-1) a^{2}}{d}\right] \frac{1}{2}-\frac{2 V_{2}}{V_{1}^{2}}\right) \frac{\log u}{u^{2}} .
$$

We can then turn our attention to the metric, for which the equation to solve is:

$$
\begin{aligned}
\dot{A} & =-\frac{1}{2(d-1)}\left(W_{0}+W_{1} \lambda+W_{2} \lambda^{2}+\ldots\right) \\
& =\frac{1}{\ell}+\frac{V_{1}}{2 \ell} \lambda+\frac{1}{\ell}\left(\frac{V_{2}}{2}-\left[1-\frac{2(d-1) a^{2}}{d}\right] \frac{V_{1}^{2}}{8}\right) \lambda^{2}+\ldots
\end{aligned}
$$


This is solved by:

$$
\begin{aligned}
A(u)= & \frac{u}{\ell}+\frac{1}{a^{2} 2(d-1) V_{1}} \log u \\
& -\frac{\ell}{a^{4} 2(d-1)^{2}}\left(\left[1-\frac{2(d-1) a^{2}}{d}\right] \frac{1}{2}-\frac{2 V_{2}}{V_{1}^{2}}\right) \frac{\log u}{u} \\
& -\frac{\ell}{a^{4}(d-1)^{2}}\left(-\frac{V_{2}}{2 V_{1}^{2}}+\left[1-\frac{2(d-1) a^{2}}{d}\right] \frac{1}{8}\right) \frac{1}{u} .
\end{aligned}
$$

Working again at next to leading order, we can also express $\lambda$ as a function of $A$ :

$$
\lambda=\frac{1}{a^{2}(d-1) V_{1} A}+\frac{1}{a^{4}(d-1)^{2} V_{1}}\left(\left[1-\frac{2(d-1) a^{2}}{d}\right] \frac{1}{2}-\frac{1}{2 V_{1}}-\frac{2 V_{2}}{V_{1}^{2}}\right) \frac{\log A}{A^{2}}
$$

Note that in this case, and unlike that of the previous section, the expansion of the potential, and the RG flow are compatible. $\lambda$ remains small during the flow.

Using the setup in section 3, where $W_{n p}$ denotes the leading non-perturbative correction to the superpotential, we are led to consider the following equation:

$$
W_{0} W_{n p}-\frac{2(d-1)}{d} W_{0}^{\prime} W_{n p}^{\prime}=0,
$$

We obtain

$$
\frac{d \log W_{n p}}{d \phi}=-\frac{d}{(d-1) a V_{1} \lambda}-\frac{d}{(d-1) a}\left(1-\frac{2 V_{2}}{V_{1}^{2}}-\frac{(d-1) a^{2}}{d}\right)+\cdots,
$$

which is integrated to

$$
W_{n p}(\lambda)=\mathcal{C} e^{-\frac{d}{(d-1) a^{2} V_{1} \lambda}} \lambda^{\frac{d}{(d-1) a^{2}}\left(1-\frac{2 V_{2}}{V_{1}^{2}}-\frac{(d-1) a^{2}}{d}\right)}+\cdots .
$$

$W_{n p}$ affects the expression for the scalar field. The equation to solve is:

$$
-\frac{\dot{\lambda}}{a \lambda}=-a\left(W_{1} \lambda+2 W_{2} \lambda^{2}\right)-\frac{d}{(d-1) a V_{1} \lambda} W_{n p}+\cdots,
$$

where we have only kept the leading non-perturbative term in (4.13). This gives:

$$
\begin{aligned}
\lambda(u)= & \frac{\ell}{a^{2}(d-1) V_{1} u}+\frac{\ell^{2}}{a^{4}(d-1)^{2} V_{1}}\left(\left[1-\frac{2(d-1) a^{2}}{d}\right] \frac{1}{2}-\frac{2 V_{2}}{V_{1}^{2}}\right) \frac{\log u}{u^{2}} \\
& -\frac{\ell}{(d-1)}\left(1+\frac{\ell}{d V_{1}^{2} u}+\frac{2 \ell^{2}}{d^{2} u^{2}}\right) \mathcal{C}_{1} e^{-\frac{d u}{\ell}}+\cdots .
\end{aligned}
$$


Finally, we compute the contribution of the non-perturbative term to the $\beta$ function. We have this time:

$$
\begin{aligned}
\beta_{\lambda} & \equiv \frac{d \lambda}{d A}=-a \frac{d \phi}{d A}=2(d-1) a \lambda \frac{d}{d \phi} \log W \\
& =2(d-1) a \lambda \frac{d}{d \phi} \log \left(W_{\text {pert }}+W_{n p}\right)=2(d-1) a \lambda \frac{d}{d \phi}\left(\log W_{\text {pert }}+\log \left[1+\frac{W_{n p}}{W_{\text {pert }}}\right]\right) .
\end{aligned}
$$

We are interested in computing the second term, namely:

$$
\begin{aligned}
& \beta_{\lambda, n p} \equiv 2(d-1) a \lambda \frac{d}{d \phi} \frac{W_{1}}{W_{0}}=\frac{\ell d \lambda}{(d-1)} W_{n p}\left[\frac{1}{V_{1} \lambda}+\frac{1}{2}-\frac{2 V_{2}}{V_{1}^{2}}-\frac{(d-1) a^{2}}{d}\right]+\cdots \cdot(4.17) \\
= & \mathcal{C} \frac{\ell d \lambda}{(d-1)}\left[\frac{1}{V_{1} \lambda}+\frac{1}{2}-\frac{2 V_{2}}{V_{1}^{2}}-\frac{(d-1) a^{2}}{d}\right] e^{-\frac{d}{(d-1) a^{2} V_{1} \lambda}} \lambda^{\frac{d}{(d-1) a^{2}}\left(1-\frac{2 V_{2}}{V_{1}^{2}}-\frac{(d-1) a^{2}}{d}\right)}+\cdots
\end{aligned}
$$

This is in agreement with general dimensional expectations.

We summarize, that this second case of marginal operators seems self-consistent and described flows similar to those of asymptotically-free gauge theories in the weakly coupled regime.

Its realization in string theory so far can be found in higher derivative contexts, several possibilities of which have been analyzed in [21], (see also [22] for a discussion of supergravity embeddings).

\section{The Multiscalar Case}

In the generic case, a single scalar operator, even if it is the only one sourced in the UV, mixes as it runs with other scalar operators. This is captured by a multi-scalar general action that we will investigate in this section.

The important difference is that apart from a bulk potential, there is a scalarfield metric now that also controls the holographic RG flow. One of our goals will be to see at what order in the scaling region the metric affects the flow.

We consider therefore the case of $N$ scalars. We start from a modified version of (2.1):

$$
S_{2 d}=M^{d-1} \int d^{d+1} x \sqrt{-g}\left\{R-\frac{1}{2} G_{i j}(\phi) \partial_{\mu} \phi^{i} \partial^{\mu} \phi^{j}+V(\phi)\right\} .
$$

The bulk equations can be easily deduced from previous calculations:

$$
\begin{gathered}
R_{\mu \nu}-\frac{1}{2} g_{\mu \nu}\left(R-\frac{1}{2} G_{i j}(\phi) \partial_{\mu} \phi^{i} \partial^{\mu} \phi^{j}+V(\phi)\right)-\frac{1}{2} G_{i j}(\phi) \partial_{\mu} \phi^{i} \partial^{\mu} \phi^{j}=0 \\
\nabla^{\mu}\left(G_{i j} \nabla_{\mu} \phi^{j}\right)+\frac{\partial V}{\partial \phi^{i}}-\frac{1}{2} \frac{\partial G_{k l}}{\partial \phi^{i}} \partial_{\mu} \phi^{k} \partial^{\mu} \phi^{l}=0
\end{gathered}
$$


From this, using the ansatz in the domain wall frame, we obtain:

$$
\begin{gathered}
d(d-1) \dot{A}^{2}+(d-1) \ddot{A}-V=0, \\
2(d-1) \ddot{A}+G_{i j} \dot{\phi}^{i} \dot{\phi}^{j}=0, \\
\Gamma^{\prime i}{ }_{j k} \dot{\phi}^{j} \dot{\phi}^{k}+\ddot{\phi}^{i}+d \dot{A} \dot{\phi}^{i}+G^{i j} \frac{\partial V}{\partial \phi^{j}}=0,
\end{gathered}
$$

where $\Gamma^{\prime}$ are the Christoffel symbols for the field metric.

A generalization of the first order equations (2.19) and (2.20) to the case of multiple scalars is:

$$
\begin{aligned}
& \dot{A}=-\frac{W}{2(d-1)} \quad, \quad \dot{\phi}^{i}=G^{i j} \frac{d W}{d \phi^{j}} \\
& \frac{d W^{2}}{2(d-1)}-G^{k l} \frac{d W}{d \phi^{k}} \frac{d W}{d \phi^{l}}=2 V .
\end{aligned}
$$

These reproduce the equations of motion (5.4), (5.5) and (5.6).

In order to compute the superpotential and the corresponding $\beta$-function, we will choose Riemann normal coordinates (RNC) around an AdS extremum. With this choice, the potential takes the following form:

$$
V(\phi)=\frac{d(d-1)}{\ell^{2}}+\frac{1}{2} M^{2}{ }_{i j} \phi^{i} \phi^{j}+g_{i j k} \phi^{i} \phi^{j} \phi^{k} \ldots .
$$

Using the properties of the RNC, we can expand the metric around the $A d S$ extremum and obtain:

$$
G_{i j}=\delta_{i j}+\left.\frac{1}{2} \phi^{k} \phi^{l} \frac{d^{2} G_{i j}}{d \phi^{k} d \phi^{l}}\right|_{\phi=0} .
$$

Expressing the second derivatives of the metric in terms of the Riemann tensor, we obtain:

$$
G^{i j}=\delta^{i j}-\frac{1}{3} \delta^{i m} \delta^{j n} R_{m k n l} \phi^{k} \phi^{l}+O\left(\phi^{3}\right) .
$$

Using the set up of the previous section, we can solve perturbatively (5.8). The superpotential $W$ takes the general form:

$$
W(\phi)=W_{0}+\sum_{i=1}^{N} W_{i} \phi^{i}+\sum_{i, j=1}^{N} W_{i j} \phi^{i} \phi^{j}+\sum_{i, j, k=1}^{N} W_{i j k} \phi^{i} \phi^{j} \phi^{k}+O\left(\phi^{4}\right)
$$

where the convention is that:

$$
W_{i_{1} \ldots i_{k}}=\left.\frac{d^{k} W}{d \phi^{i_{1}} \cdots d \phi^{i_{k}}}\right|_{\phi=0}
$$


As in the single scalar case, we should put the first derivatives to zero, in order for the vanishing of the scalars to happen at the extremum. Solving perturbatively the equation (5.8) for the superpotential, we obtain the following set of equations:

$$
\begin{aligned}
& \frac{d}{2(d-1)} W_{0}^{2}=\frac{2 d(d-1)}{\ell^{2}} \\
& \frac{d}{(d-1)} W_{0} W_{i j}-\delta^{k l} 4 W_{j k} W_{i l}=M_{i j}^{2} \\
& \frac{d}{(d-1)} W_{0} \sum_{i, j, p=1}^{N} W_{i j p} \phi^{i} \phi^{j} \phi^{p}-12 \delta^{k l} \sum_{p=1}^{N} W_{p k} \phi^{p} \sum_{i, j=1}^{N} W_{i j l} \phi^{i} \phi^{j}=2 g_{i, j, k} \phi^{i} \phi^{j} \phi^{k}
\end{aligned}
$$

which can be simplified to:

$$
\begin{aligned}
& W_{0}=-\frac{2(d-1)}{\ell} \\
& -\frac{2 d W_{i j}}{\ell}-\delta^{k l} 4 W_{i l} W_{k j}=M_{i j}^{2} \\
& -\frac{2 d}{\ell} W_{i j p}-12 \delta^{k l} W_{k(p} W_{i j) l}=2 \delta_{n, 3} g_{i j p}
\end{aligned}
$$

where indices are raised and lowered using the flat Euclidean metric since all quantities are evaluated at the center of the RNC, and where the sign of $W_{0}$ is chosen in order to recover an $A d S$ solution when all the scalar fields vanish.

Without loss of generality, we can take $M_{i j}^{2}$ and $W_{i j}$ to be real and symmetric. It is then straightforward to show using (5.18) that $\left[M^{2}, W\right]=0$. Hence there exists a coordinate system in the scalar fields space in which both these matrices can be simultaneously diagonalized by a constant $O(N)$ rotation. With this choice, we can write:

$$
M^{2}=\operatorname{diag}\left(m_{1}^{2}, m_{2}^{2}, \ldots, m_{N}^{2}\right) .
$$

The eigenvalues are real, and some of them can be equal to zero, in which case this would correspond to a marginal operator. Denoting $w_{i}$ the eigenvalues of $W_{i j}$, the equation (5.18) can be written as:

$$
4 w_{i}^{2}+\frac{2 d}{\ell} w_{i}+m_{i}^{2}=0
$$

which is solved by:

$$
w_{i}=\frac{-d \pm \sqrt{d^{2}-4 \ell^{2} m_{i}^{2}}}{4 \ell} .
$$

We now can define the holographic version of the $\beta$-function for the case of multiple scalars:

$$
\beta_{\phi}^{i} \equiv \frac{d \phi^{i}}{d A}=-2(d-1) G^{i j} \frac{d}{d \phi^{j}} \log W
$$


Using (5.11), we obtain:

$$
\begin{aligned}
\beta_{\phi}^{i}= & 2 \ell w_{i} \phi^{i}+3 \ell \sum_{p, q=1}^{N} W_{i p q} \phi^{p} \phi^{q} \\
& +\sum_{p, q, r=1}^{N}\left(4 \ell W_{i p q r}+\frac{\ell^{2}}{(d-1)} w_{p} w_{i} \delta^{p q} \delta^{i r}-\frac{2 \ell}{3} w_{r} R_{i p r q}\right) \phi^{p} \phi^{q} \phi^{r}+O\left(\phi^{4}\right) .
\end{aligned}
$$

From this last equation and (5.17), (5.18) and (5.19), we conclude the following

- If a subset of operators are marginal, then their leading $\beta$-function starts generically at one-loop.

- The mixing with relevant operators is also happening first at the one-loop. This persists even when all operators are marginal.

- The field space metric (Zamolodchikov metric), equivalent to the curvature of the field space first affects the $\beta$-functions at two-loop level.

\section{Acknowledgements}

We would like to thank J. Gauntlet, N. Warner and K. Pilch for correspondence.

This work was supported in part by European Union's Seventh Framework Programme under grant agreements (FP7-REGPOT-2012-2013-1) no 316165, PIF-GA2011-300984, the EU program "Thales" MIS 375734, by the European Commission under the ERC Advanced Grant BSMOXFORD 228169and was also co-financed by the European Union (European Social Fund, ESF) and Greek national funds through the Operational Program "Education and Lifelong Learning" of the National Strate-

gic Reference Framework (NSRF) under "Funding of proposals that have received a positive evaluation in the 3rd and 4th Call of ERC Grant Schemes". The research leading to these results has also received funding from the People Programme (Marie Curie Actions) of the European Union's Seventh Framework Programme FP7/20072013/ under REA Grant Agreement No 317089 (GATIS). 


\section{Appendices}

\section{A. Perturbative Calculations for $n=3,4,5$.}

A.1 $n=3$

$$
\begin{aligned}
W(\phi)= & -\frac{2(d-1)}{\ell}-\frac{g_{3}}{d \ell} \phi^{3}-\frac{9 g_{3}^{2}}{2 d^{3} \ell} \phi^{4}-\frac{54 g_{3}^{3}}{d^{5} \ell} \phi^{5}+g_{3}^{2} \frac{d^{5}+3888(1-d) g_{3}^{2}}{4(d-1) d^{7} \ell} \phi^{6} \\
& +27 g_{3}^{3} \frac{d^{5}+3312(1-d) g_{3}^{2}}{4(d-1) d^{9} \ell} \phi^{7}+27 g_{3}^{4} \frac{119 d^{5}+362016(1-d) g_{3}^{2}}{16(d-1) d^{11} \ell} \phi^{8} \\
& -\frac{g_{3}^{3}}{8(d-1)^{2} d^{13} \ell}\left(d^{10}+53460 d^{5}(1-d) g_{3}^{2}-304850304 d g_{3}^{4}+152425152\left(1+d^{2}\right) g_{3}^{4}\right) \phi^{9} \\
& -\frac{27 g_{3}^{4}}{16(d-1)^{2} d^{15} \ell}\left(5 d^{10}+145152 d^{5}(1-d) g_{3}^{2}+392097024\left(1+d^{2}\right) g_{3}^{4}-784194048 d g_{3}^{4}\right) \phi^{10}
\end{aligned}
$$

$$
\begin{aligned}
\beta_{\phi}= & -\frac{3 g_{3}}{d} \phi^{2}-\frac{18 g_{3}^{2}}{d^{3}} \phi^{3}-\frac{270 g_{3}^{3}}{d^{5}} \phi^{4}+3 g_{3}^{2} \frac{d^{5}+1944(1-d) g_{3}^{2}}{(d-1) d^{7}} \phi^{5}+63 g_{3}^{3} \frac{d^{5}+2484(1-d) g_{3}^{2}}{(d-1) d^{9}} \phi^{6} \\
& +81 \frac{23 d^{5} g_{3}^{4}+60336(1-d) g_{3}^{6}}{(d-1) d^{11}} \phi^{7} \\
& -3 g_{3}^{3} \frac{d^{10}+21870 d^{5}(1-d) g_{3}^{2}+57159432\left(1+d^{2}\right) g_{3}^{4}-114318864 d g_{3}^{4}}{(d-1)^{2} d^{13}} \phi^{8} \\
& -135 g_{3}^{4} \frac{d^{10}+19188 d^{5}(1-d) g_{3}^{2}+49012128\left(1+d^{2}\right) g_{3}^{4}-98024256 d g_{3}^{4}}{(d-1)^{2} d^{15}} \phi^{9}
\end{aligned}
$$

A.2 $n=4$

$$
\begin{aligned}
W(\phi)= & -\frac{2(d-1)}{\ell}-\frac{g_{4}}{d \ell} \phi^{4}-\frac{8 g_{4}^{2}}{d^{3} \ell} \phi^{6}+g_{4}^{2} \frac{d^{3}+768(1-d) g_{4}}{4(d-1) d^{5} \ell} \phi^{8}+12 g_{4}^{3} \frac{d^{3}+608(1-d) g_{4}}{(d-1) d^{7} \ell} \phi^{10} \\
& -g_{4}^{3} \frac{d^{6}+5504 d^{3}(1-d) g_{4}+2924544\left(1+d^{2}\right) g_{4}^{2}-5849088 d g_{4}^{2}}{8(d-1)^{2} d^{9} \ell} \phi^{12} \\
& -3 g_{4}^{4} \frac{5 d^{6}+15424 d^{3}(1-d) g_{4}+7409664\left(1+d^{2}\right) g_{4}^{2}-14819328 d g_{4}^{2}}{(d-1)^{2} d^{11} \ell} \phi^{14} \\
& +\frac{g_{4}^{4}}{64(d-1)^{3} d^{13} \ell}\left[5 d^{9}+100352 d^{6}(1-d) g_{4}-452984832 d^{4} g_{4}^{2}\right. \\
& +226492416 d^{3}\left(1+d^{2}\right) g_{4}^{2}+100317265920\left(1-d^{3}\right) g_{4}^{3} \\
& \left.-300951797760 d(1-d) g_{4}^{3}\right] \phi^{16}
\end{aligned}
$$




$$
\begin{aligned}
\beta_{\phi}= & -\frac{4 g_{4}}{d} \phi^{3}-\frac{48 g_{4}^{2}}{d^{3}} \phi^{5}+4 g_{4}^{2} \frac{d^{3}+384(1-d) g_{4}}{d^{5}(d-1)} \phi^{7}+160 g_{4}^{3} \frac{d^{3}+456(1-d) g_{4}}{(d-1) d^{7}} \phi^{9} \\
& -4 g_{4}^{3} \frac{d^{6}+2400 d^{3}(1-d) g_{4}+1096704\left(1+d^{2}\right) g_{4}^{2}-2193408 d g_{4}^{2}}{(d-1)^{2} d^{9}} \phi^{11} \\
& -336 g_{4}^{4} \frac{d^{6}+2112 d^{3}(1-d) g_{4}+926208\left(1+d^{2}\right) g_{4}^{2}-1852416 d g_{4}^{2}}{(d-1)^{2} d^{11}} \phi^{13} \\
& +\frac{4 g_{4}^{4}}{(d-1)^{3} d^{13}}\left[d^{9}+8192 d^{6}(1-d) g_{4}-30081024 d^{4} g_{4}^{2}+15040512 d^{3}\left(1+d^{2}\right) g_{4}^{2}\right. \\
& \left.+6269829120\left(1-d^{3}\right) g_{4}^{3}-18809487360 d g_{4}^{3}(1-d)\right] \phi^{15}
\end{aligned}
$$

A.3 $n=5$

$$
\begin{aligned}
W(\phi)= & -\frac{2(d-1)}{\ell}-\frac{g_{5}}{d \ell} \phi^{5}-\frac{25 g_{5}^{2}}{2 d^{3} \ell} \phi^{8}+\frac{g_{5}^{2}}{4(d-1) d^{2} \ell} \phi^{10} \\
& -\frac{500 g_{5}^{3}}{d^{5} \ell} \phi^{11}+\frac{75 g_{5}^{3}}{4(d-1) d^{4} \ell} \phi^{13}-\frac{32500 g_{5}^{4}}{d^{7} \ell} \phi^{14}-\frac{g_{5}^{3}}{8(d-1)^{2} d^{3} \ell} \phi^{15} \\
& +\frac{28125 g_{5}^{4}}{16(d-1) d^{6} \ell} \phi^{16}-\frac{2825000 g_{5}^{5}}{d^{9} \ell} \phi^{17}-\frac{375 g_{5}^{4}}{16(d-1)^{2} d^{5} \ell} \phi^{18}+\frac{198125 g_{5}^{5}}{(d-1) d^{8} \ell} \phi^{19} \\
& +5 g_{5}^{4} \frac{d^{7}+3849600000\left(1-d^{3}\right) g_{5}^{2}-11548800000 d(1-d) g_{5}^{2}}{64(d-1)^{3} d^{11} \ell} \phi^{20} \\
\beta_{\phi}= & -\frac{5 g_{5}}{d} \phi^{4}-\frac{100 g_{5}^{2}}{d^{3}} \phi^{7}+\frac{5 g_{5}^{2}}{(d-1) d^{2}} \phi^{9}-\frac{5500 g_{5}^{3}}{d^{5}} \phi^{10}+\frac{325 g_{5}^{3}}{(d-1) d^{4}} \phi^{12} \\
& -\frac{455000 g_{5}^{4}}{d^{7}} \phi^{13}-\frac{5 g_{5}^{3}}{(d-1)^{2} d^{3}} \phi^{14}+\frac{32750 g_{5}^{4}}{(d-1) d^{6}} \phi^{15}-\frac{48025000 g_{5}^{5}}{d^{9}} \phi^{16} \\
& -\frac{675 g_{5}^{4}}{(d-1)^{2} d^{5}} \phi^{17}+\frac{4132500 g_{5}^{5}}{(d-1) d^{8}} \phi^{18} \\
& +5 g_{5}^{4} \frac{d^{7}+1203000000\left(1-d^{3}\right) g_{5}^{2}-3609000000 d(1-d) g_{5}^{2}}{(d-1)^{3} d^{11}} \phi^{19}
\end{aligned}
$$




\section{References}

[1] E. Kiritsis, "Lorentz violation, Gravity, Dissipation and Holography," JHEP 1301 (2013) 030 [ArXiv:1207.2325][hep-th].

[2] E. Kiritsis, "On novel string theories from 4d gauge theories," [ArXiv:1301.6810][hep-th].

[3] S. -S. Lee, "Quantum Renormalization Group and Holography," [ArXiv:1305.3908][hep-th].

[4] J. de Boer, E. P. Verlinde and H. L. Verlinde, "On the holographic renormalization group," JHEP 0008 (2000) 003 ArXiv:hep-th/9912012.

[5] I. Papadimitriou and K. Skenderis, "Correlation functions in holographic RG flows," JHEP 0410, 075 (2004) [ArXiv:hep-th/0407071];

I. Papadimitriou, "Holographic Renormalization of general dilaton-axion gravity," JHEP 1108 (2011) 119 [ArXiv:1106.4826][hep-th].

[6] M. Bianchi, D. Z. Freedman and K. Skenderis, "How to go with an RG flow," JHEP 0108, 041 (2001) ArXiv:hep-th/0105276.

[7] D. Nickel, D. T. Son, "Deconstructing holographic liquids," [ArXiv:1009.3094][hep-th];

I. Heemskerk, J. Polchinski, "Holographic and Wilsonian Renormalization Groups," [ArXiv:1010.1264][hep-th];

T. Faulkner, H. Liu, M. Rangamani, "Integrating out geometry: Holographic

Wilsonian RG and the membrane paradigm," [ArXiv:1010.4036][hep-th].

[8] C. Charmousis, B. Goutéraux, B. S. Kim, E. Kiritsis and R. Meyer, "Effective

Holographic Theories for low-temperature condensed matter systems," JHEP 1011

(2010) 151 [ArXiv:1005.4690] [hep-th];

B. Gouteraux and E. Kiritsis, "Generalized Holographic Quantum Criticality at Finite Density," JHEP 1112 (2011) 036 [ArXiv:1107.2116][hep-th].

[9] E. Kiritsis and V. Niarchos, "The holographic quantum effective potential at finite temperature and density," JHEP 1208 (2012) 164 [ArXiv:1205.6205][hep-th].

[10] O. Aharony, S. S. Gubser, J. M. Maldacena, H. Ooguri and Y. Oz, "Large $N$ field theories, string theory and gravity", Phys. Rept. 323 (2000) 183-386, [ArXiv:9905111][hep-th];

E. D'Hoker and D. Z. Freedman, "Supersymmetric gauge theories and the AdS/CFT correspondence", [ArXiv:0201253][hep-th].

[11] E. Kiritsis, W. Li, F. Nitti, to appear.

[12] U. Gursoy and E. Kiritsis, "Exploring improved holographic theories for QCD: Part I," JHEP 0802 (2008) 032 [ArXiv:0707.1324][hep-th]; 
U. Gursoy, E. Kiritsis and F. Nitti, "Exploring improved holographic theories for QCD: Part II," JHEP 0802 (2008) 019 [ArXiv:0707.1349/[hep-th]].

[13] M. C. N. Cheng, S. A. Hartnoll and C. A. Keeler, "Deformations of Lifshitz holography," JHEP 1003 (2010) 062 [ArXiv:0912.2784][hep-th].

[14] E. Kiritsis, "Asymptotic freedom, asymptotic flatness and cosmology," |ArXiv:1307.5873][hep-th].

[15] A. A. Starobinsky, "A New Type of Isotropic Cosmological Models Without Singularity," Phys. Lett. B 91 (1980) 99.

[16] E. Kiritsis, "String theory in a nutshell," Princeton University Press, 2007

[17] L. Girardello, M. Petrini, M. Porrati and A. Zaffaroni, "Novel local CFT and exact results on perturbations of $N=4$ superYang Mills from AdS dynamics," JHEP 9812 (1998) 022 [ArXiv:hep-th/9810126].

[18] U. Gursoy, E. Kiritsis, L. Mazzanti and F. Nitti, "Holography and Thermodynamics of 5D Dilaton-gravity," JHEP 0905 (2009) 033 [ArXiv:0812.0792][hep-th].

[19] T. Faulkner, G. T. Horowitz and M. M. Roberts, "New stability results for Einstein scalar gravity," Class. Quant. Grav. 27, 205007 (2010) [ArXiv:1006.2387][hep-th].

[20] U. Gursoy, E. Kiritsis, L. Mazzanti, G. Michalogiorgakis and F. Nitti, "Improved Holographic QCD," Lect. Notes Phys. 828 (2011) 79 [ArXiv:1006.5461][hep-th];

[21] E. Kiritsis, "Dissecting the string theory dual of QCD," Fortsch. Phys. 57 (2009) 396 [ArXiv:0901.1772][hep-th].

[22] F. Farakos, A. Kehagias and A. Riotto, "On the Starobinsky Model of Inflation from Supergravity," Nucl. Phys. B 876 (2013) 187 [ArXiv:1307.1137][hep-th];

J. Ellis, D. V. Nanopoulos and K. A. Olive, "No-Scale Supergravity Realization of the Starobinsky Model of Inflation," Phys. Rev. Lett. 111 (2013) 111301

[ArXiv:1305.1247][hep-th]. 